\title{
Logic-gated Cell-derived Nanovesicles via DNA-based Smart Recognition Module
}

Huidong Huang ${ }^{1}$, Zhenzhen Guo ${ }^{1}$, Chunjuan Zhang ${ }^{1}$, Cheng Cui ${ }^{1}$, Ting Fu², Qiaoling Liu ${ }^{1 *}$, Weihong Tan ${ }^{1,2,3 *}$

${ }^{1}$ Molecular Science and Biomedicine Laboratory (MBL), State Key Laboratory of Chemo/Biosensing and Chemometrics, College of Biology, College of Chemistry and Chemical Engineering, Aptamer Engineering Center of Hunan Province, Hunan University, Changsha, Hunan 410082, China

${ }^{2}$ The Cancer Hospital of the University of Chinese Academy of Sciences (Zhejiang Cancer Hospital), Institute of Basic Medicine and Cancer (IBMC), Chinese Academy of Sciences, Hangzhou, Zhejiang 310022, China

${ }^{3}$ Institute of Molecular Medicine (IMM), Renji Hospital, Shanghai Jiao Tong University School of Medicine, Shanghai Jiao Tong University, Shanghai 200240, China.

*Authors for correspondence:

Prof. Weihong Tan

Email: tan@hnu.edu.cn

Dr. Qiaoling Liu

Email: qlliu@iccas.ac.cn 
Table S1: Single stand DNA sequences used in the experiment.

\begin{tabular}{|c|c|}
\hline Name & DNA Sequences $\left(5^{\prime}-3^{\prime}\right)$ \\
\hline S1 & $\begin{array}{c}\text { ACATTCCTAAGTCTGAAACATTACAGCTTGCTACACGAGAAG } \\
\text { AGCCGCCATAGTATTTTTTTTTTGTATCCAGTGGCTCAGACTT } \\
\text { TTGAC }\end{array}$ \\
\hline S2 & $\begin{array}{l}\text { TATCACCAGGCAGTTGACAGTGTAGCAAGCTGTAATAGATGC } \\
\text { GAGGGTCCAATAC }\end{array}$ \\
\hline $\begin{array}{l}\text { Alexa } \\
\text { Fluor } \\
488-\mathrm{S} 2\end{array}$ & $\begin{array}{c}\text { Alexa Fluor } \\
\text { 488-TATCACCAGGCAGTTGACAGTGTAGCAAGCTGTAATAGA } \\
\text { TGCGAGGGTCCAATAC }\end{array}$ \\
\hline S3 & $\begin{array}{c}\text { TCAACTGCCTGGTGATAAAACGACACTACGTGGGAATCTACT } \\
\text { ATGGCGGCTCTTC }\end{array}$ \\
\hline S4 & $\begin{array}{l}\text { TTCAGACTTAGGAATGTGCTTCCCACGTAGTGTCGTTTGTATT } \\
\text { GGACCCTCGCAT }\end{array}$ \\
\hline $\mathrm{cSgc} 8$ & $\begin{array}{c}\text { ATCTAACTGCTGCGCCGCCGGGAAAATACTGTACGGTTAGAT } \\
\text { TTTTTGTCAAAAGTCTGAGCCACTGGATAC }\end{array}$ \\
\hline S2-chol & $\begin{array}{l}\text { TATCACCAGGCAGTTGACAGTGTAGCAAGCTGTAATAGATGC } \\
\text { GAGGGTCCAATACTT-Cholesterol }\end{array}$ \\
\hline S3-chol & $\begin{array}{c}\text { TCAACTGCCTGGTGATAAAACGACACTACGTGGGAATCTACT } \\
\text { ATGGCGGCTCTTCTT- Cholesterol }\end{array}$ \\
\hline S4-chol & $\begin{array}{l}\text { TTCAGACTTAGGAATGTGCTTCCCACGTAGTGTCGTTTGTATT } \\
\text { GGACCCTCGCATTT- Cholesterol }\end{array}$ \\
\hline $\mathrm{I}-17$ & $\begin{array}{c}\text { CCCCCCAATCCCCCCTGTATTTTCCCGGTCCCCCCAATCCCCC } \\
\mathrm{C}\end{array}$ \\
\hline $\mathrm{I}-19$ & $\begin{array}{c}\text { CCCCCCAATCCCCCACTGTATTTTCCCGGTCCCCCCAATCCCC } \\
\text { CC }\end{array}$ \\
\hline I-19.6 & $\begin{array}{c}\text { CCCCCCAATCCCCCCTGTATTTTCCCGGCTCCCCCCAATCCCC } \\
\text { CC }\end{array}$ \\
\hline $\mathrm{I}-21$ & $\begin{array}{c}\text { CCCCCCAATCCCCCACTGTATTTTCCCGGCTCCCCCCAATCCC } \\
\text { CCC }\end{array}$ \\
\hline
\end{tabular}




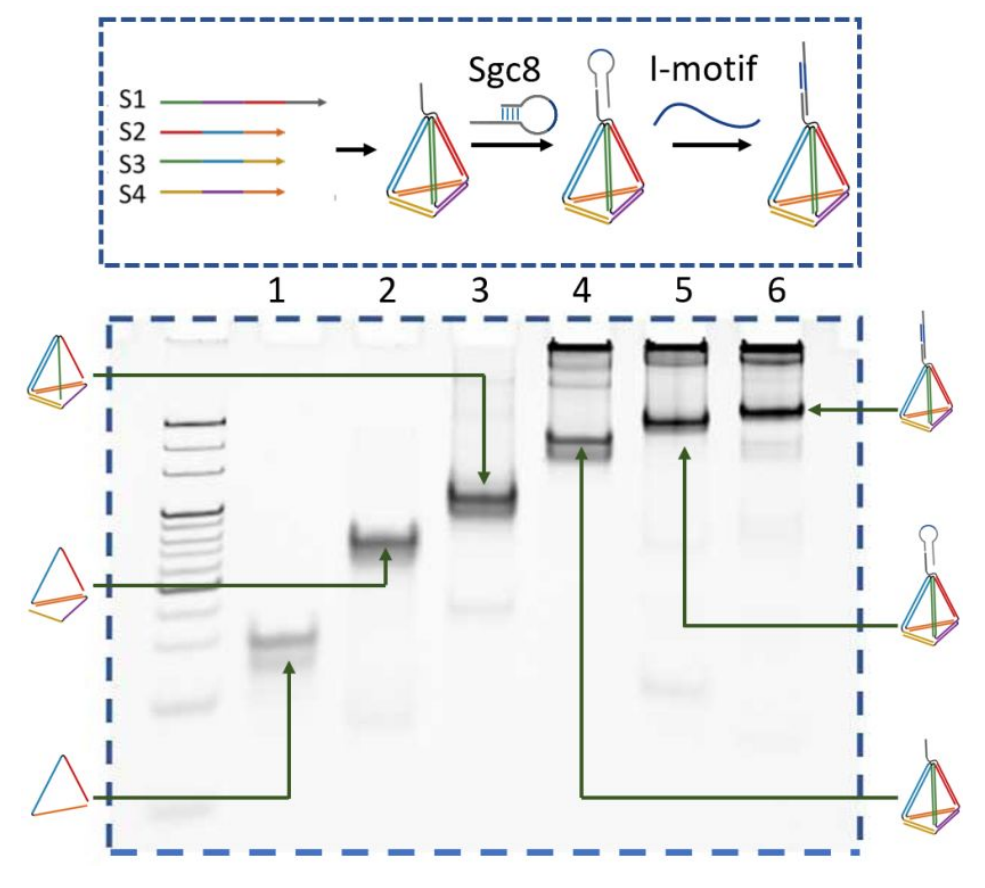

Figure S1. Characterization of DNA logic gate by $5 \%$ native polyacrylamide gel electrophoresis. Lane 1, S1; lane 2, S1+S2; lane 3, S1+S2+S3; lane 4, S1+S2+S3+S4; lane 5, S1+S2+S3+S4+cSgc8; lane 6, S1+S2+S3+S4+cSgc8+I-21. 

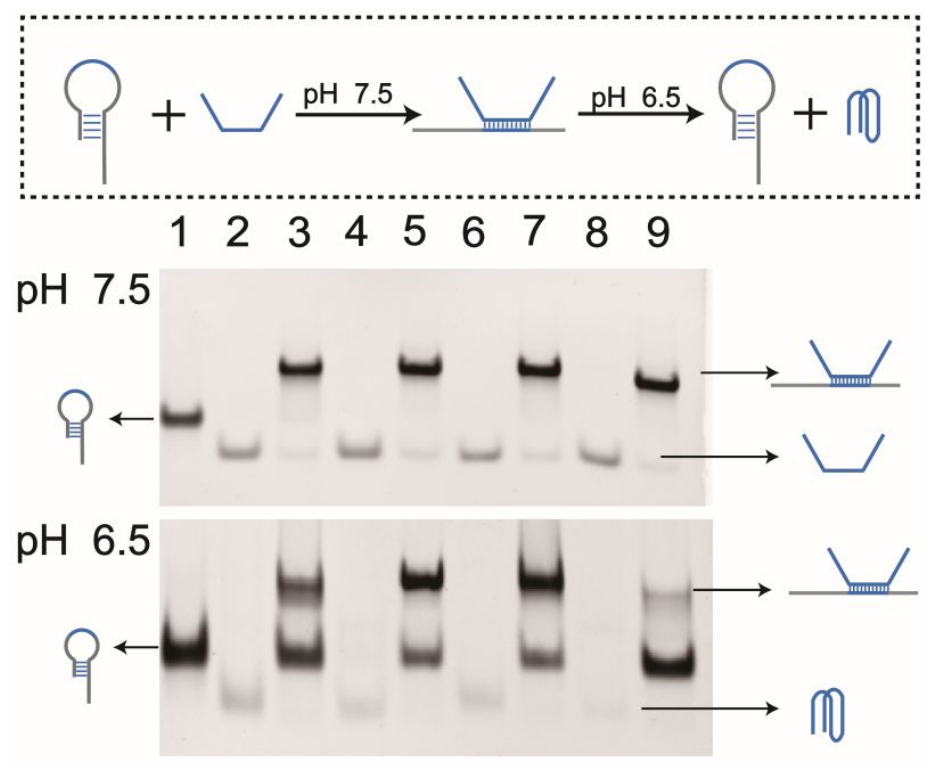

Figure S2. Optimize the sequence of I-motif for the construction of $\mathrm{pH}$-responsive cSgc8/I-motif module by $10 \%$ native polyacrylamide gel electrophoresis. The optimal pH responsiveness is observed using I-motif 21 sequence. Lane 1, cSgc8; lane 2, I-motif 17; lane 3, I-motif $17+\mathrm{cSgc} 8$; lane 4, I-motif 19; lane5, I-motif $19+\mathrm{cSgc} 8$ lane 6, I-motif 19.6; lane 7, I-motif 19.6 + cSgc8, lane 8: I-motif 21, lane 9: I-motif 21 $+$ cSgc8. 

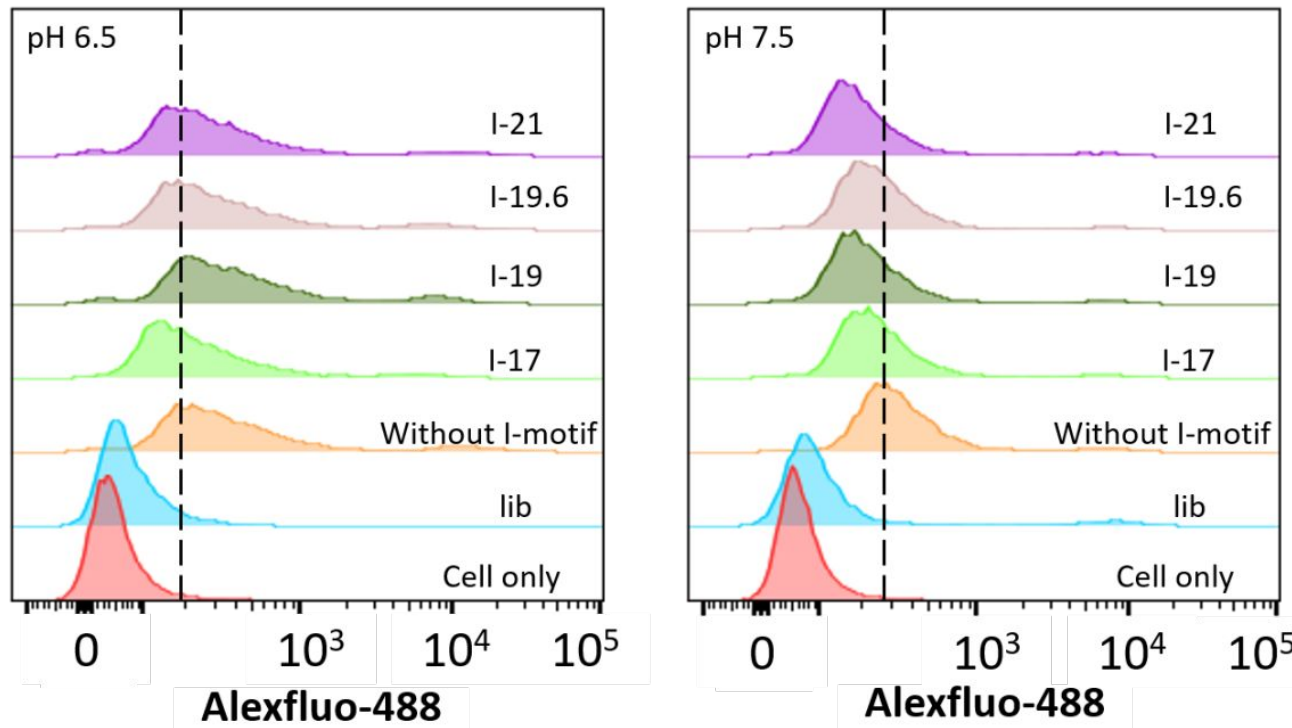

Figure S3. Flow cytometry analysis of HCT116 cells incubated with 250 nM DNA logic-gated modules in buffer solution at $\mathrm{pH} 6.5$ (left) or $\mathrm{pH} 7.5$ (right). DNA logic-gated modules consisting of I-21, I-19.6, I-19, I-17 or lib show different recognition capability to cells (from top to bottom). About 10000 events were counted for each sample. 


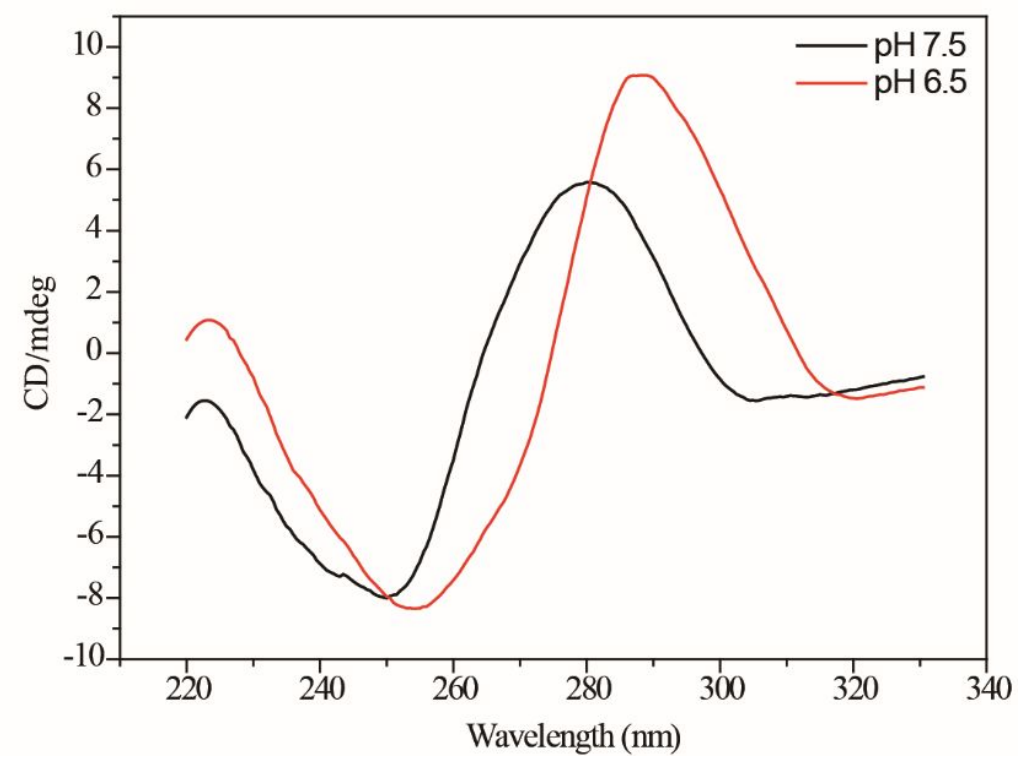

Figure S4. The CD spectra of the Sgc8/I-motif 21 hybridization complex $(10 \mu \mathrm{M})$ in PBS buffer at either pH 6.5 (red line) or pH 7.5 (black line). 

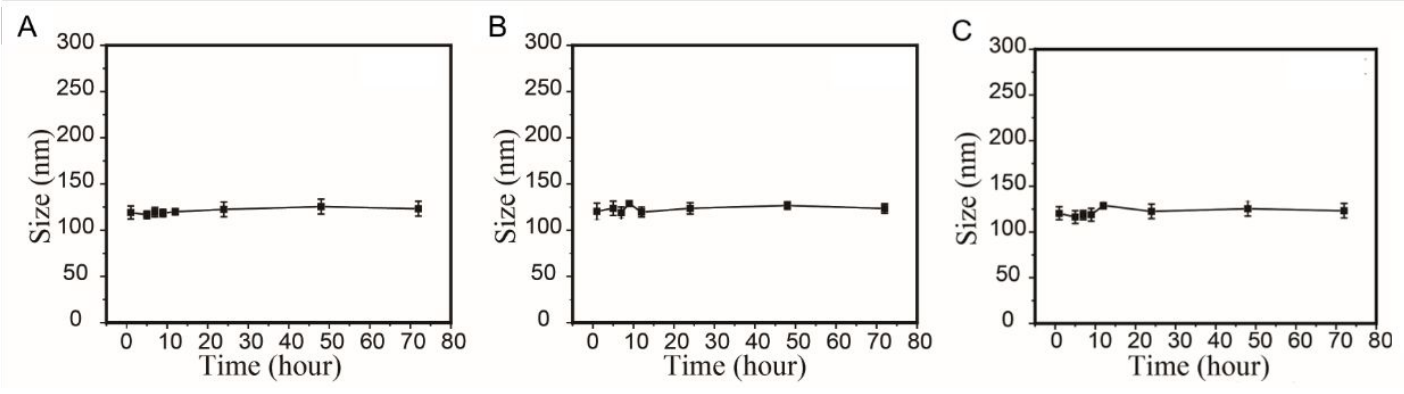

Figure S5. The particle size of nanovesicles in DPBS (A), 1640 (B), DMEM (C) respectively stored at $4{ }^{\circ} \mathrm{C}$ for different time interval ( 1 hour $/ 3$ hours $/ 5$ hours $/ 7$ hours/9 hours/24 hours/48 hours/72 hours). 


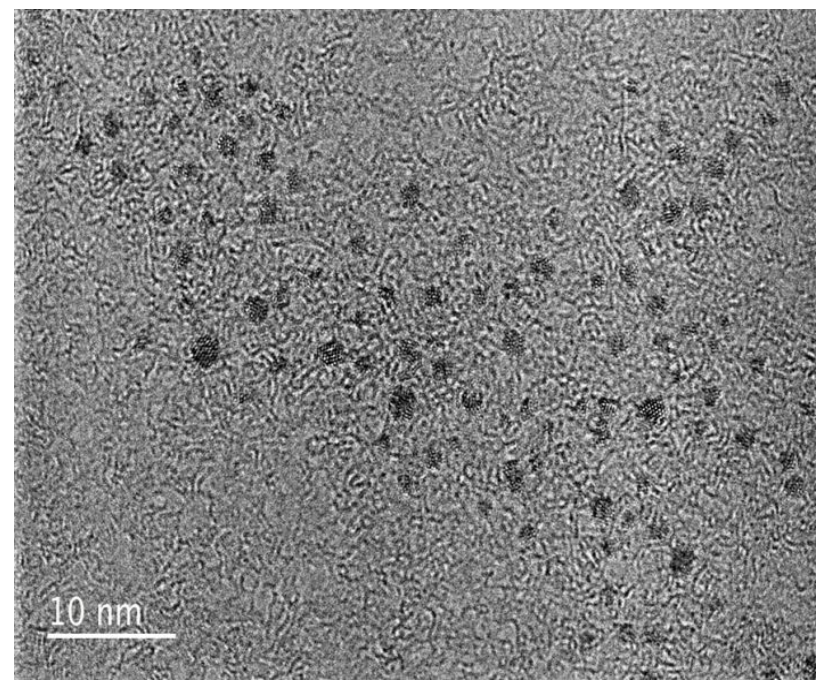

Figure S6. TEM image of GCDs. The averaged particle size of GCDs is about $2 \mathrm{~nm}$. 


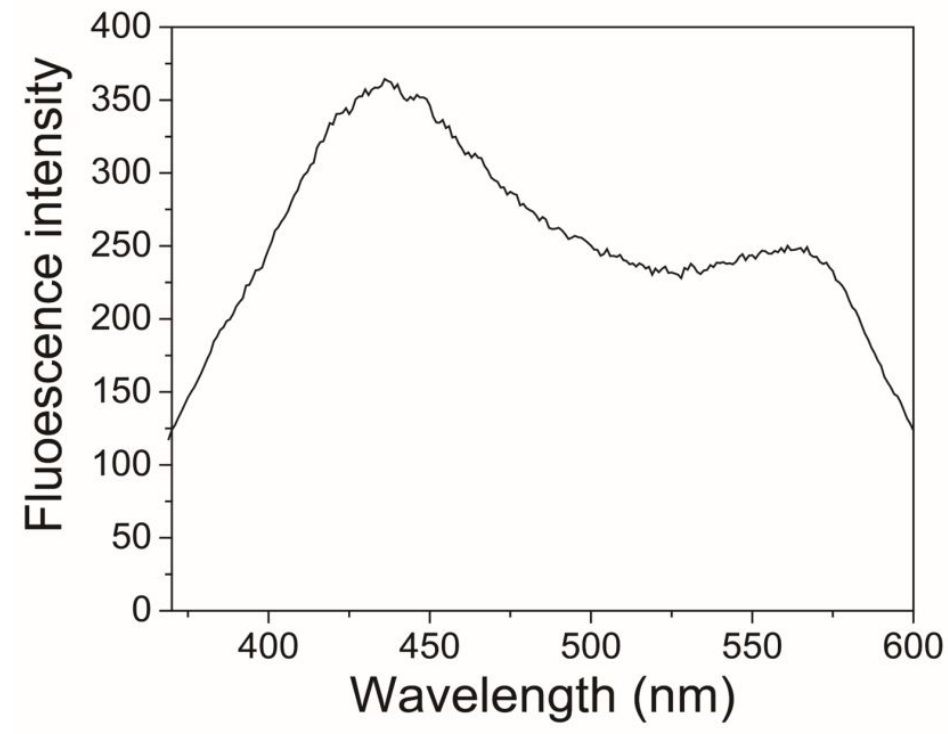

Figure S7. Fluorescence spectrum of GCDs in double distilled water under excitation at $350 \mathrm{~nm}$. Two distinctive emission peaks at 450 and $570 \mathrm{~nm}$ show the characteristic

spectrum

of

GCDs. 


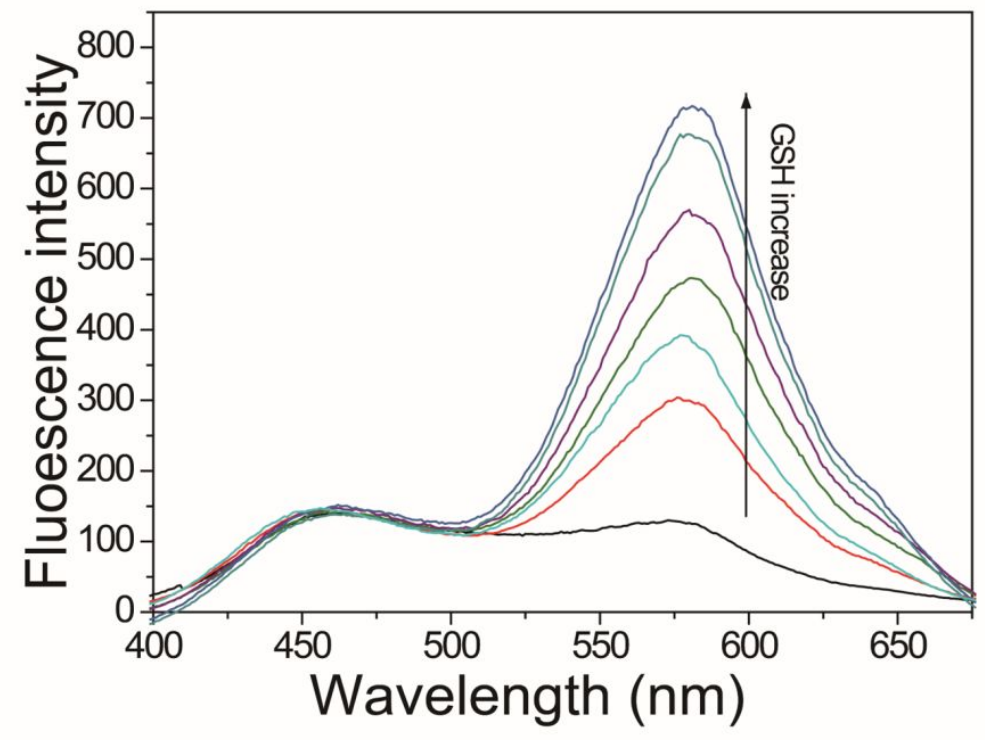

Figure S8. Fluorescence intensity of GCDs in the presence of different concentration of GSH from $1 \mathrm{mM}$ to $12 \mathrm{mM}$. GCDs in double distilled water under excitation at 350 $\mathrm{nm}$. 


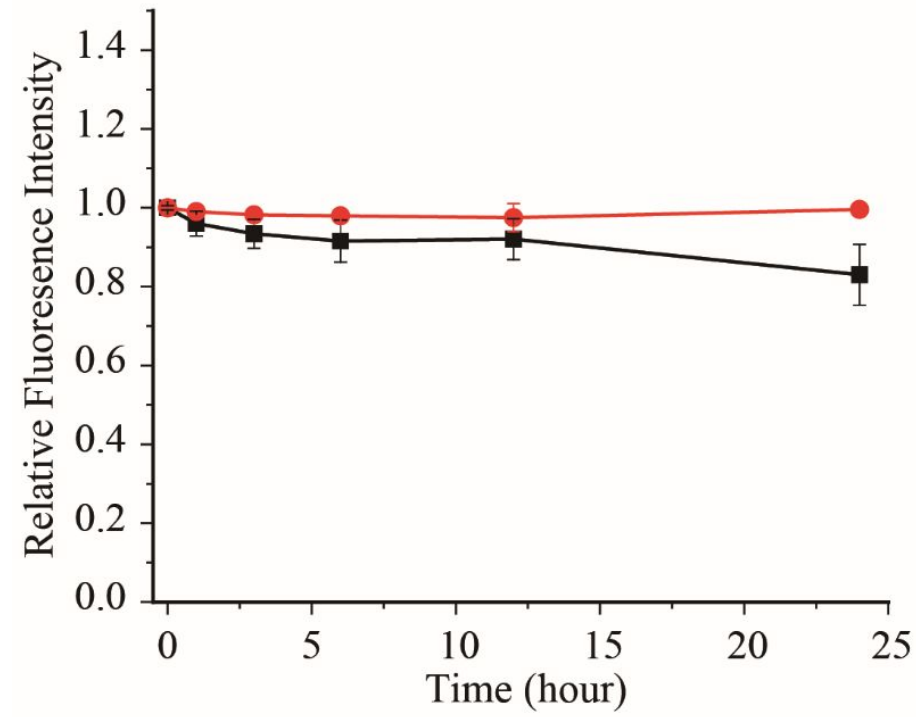

Figure S9. Relative fluorescence intensity of nanovesicles encapsulated with GCDs during the storage at $4{ }^{\circ} \mathrm{C}$. Circle, naked GCDs in DPBS medium; Square, GCDs encapsulated within nanovesicles. 


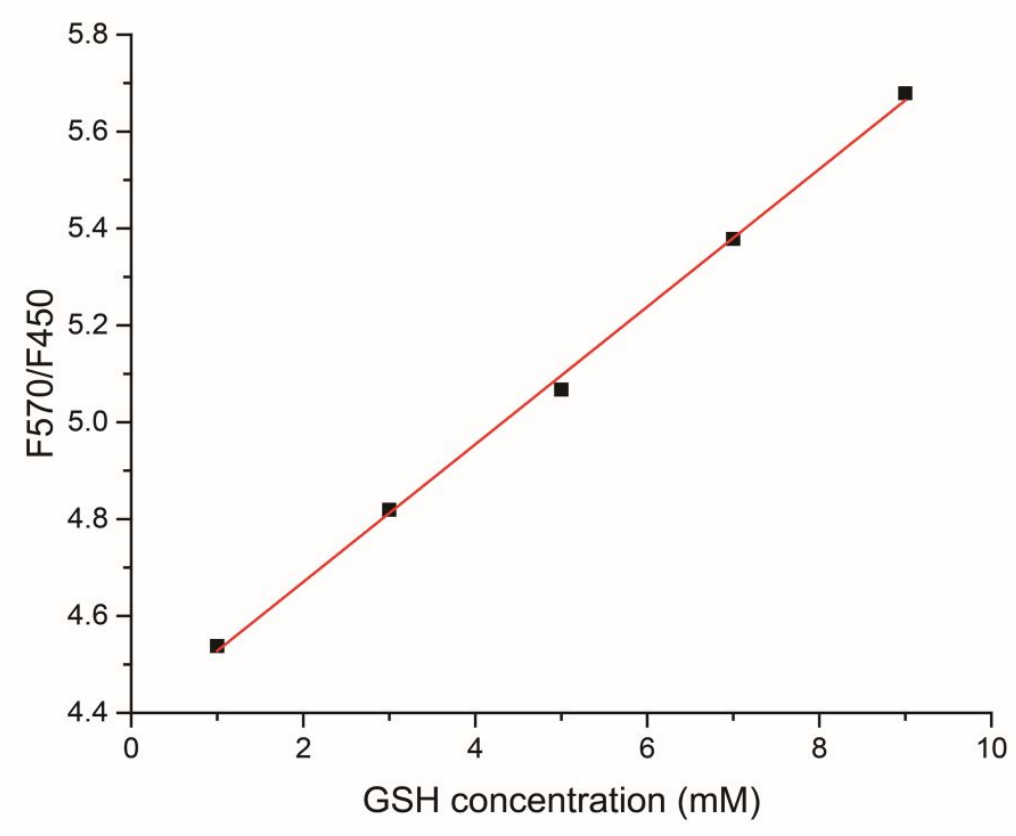

Figure S10. Fluorescence ratio $(\Delta \mathrm{F} 570 / \mathrm{F} 450)$ of GCDs upon the addition of different concentration of GSH. The equation of calibration curve was $\Delta \mathrm{F} 570 / \mathrm{F} 435=4.38602$ $+0.14207 \mathrm{c}$ with a linear coefficient $\mathrm{R}^{2}=0.9980$ GCDs in double distilled water under excitation at $350 \mathrm{~nm}$. 

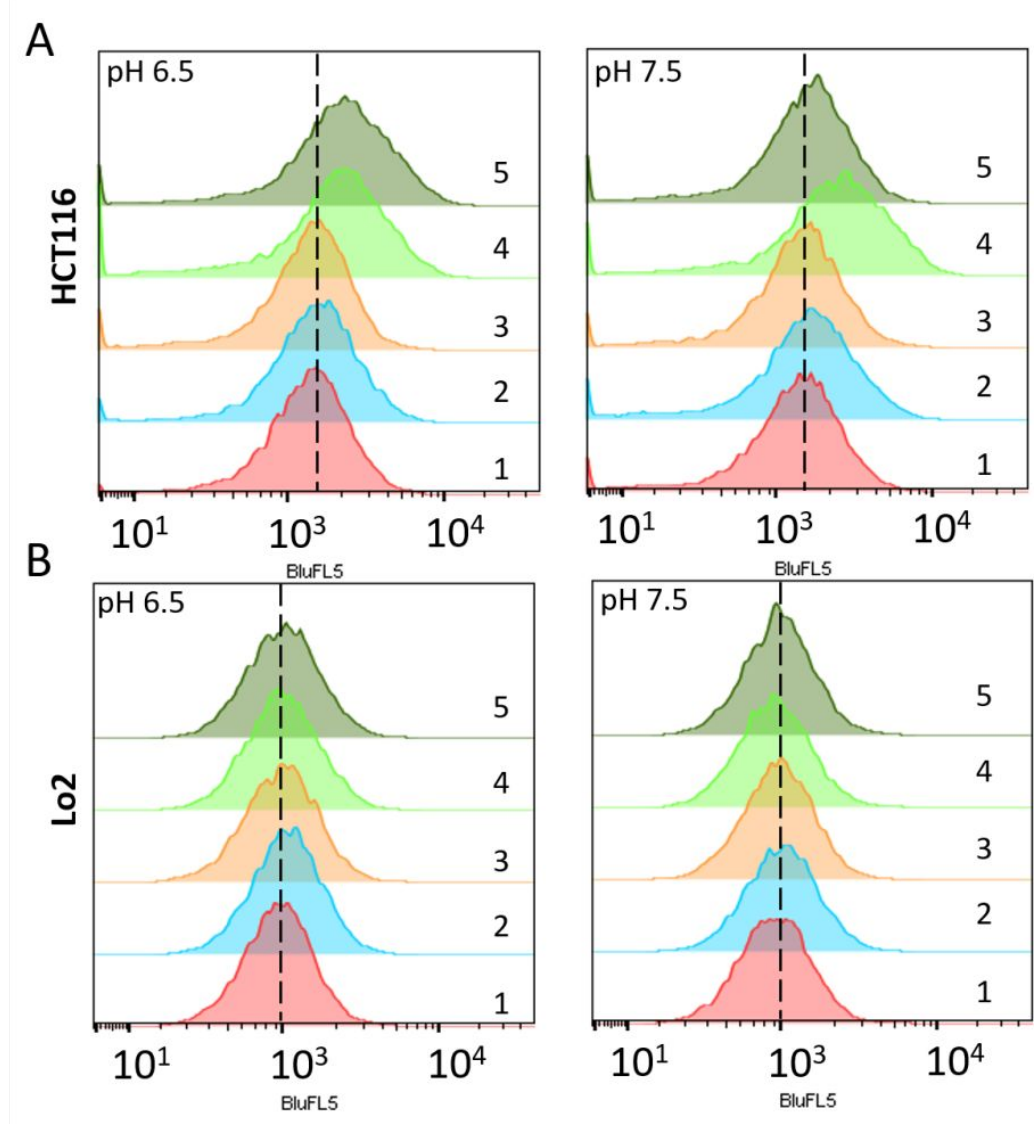

Figure S11. Flow cytometry analysis of HCT116 cells (A) and Lo2 cells (B) incubated with nanovesicles modified with various DNA logic gate in buffer solution at different $\mathrm{pH}$ values. 1, cell only; 2, cells incubated with GCDs loaded nanovesicles; 3, cells incubated with lib-T modified GCDs loaded nanovesicles; 4, cells incubated with cSgc8-T modified GCDs loaded nanovesicles; 5, cells incubated with I21-cSgc8-T modified GCDs loaded nanovesicles. About 10000 events were counted for each sample. 


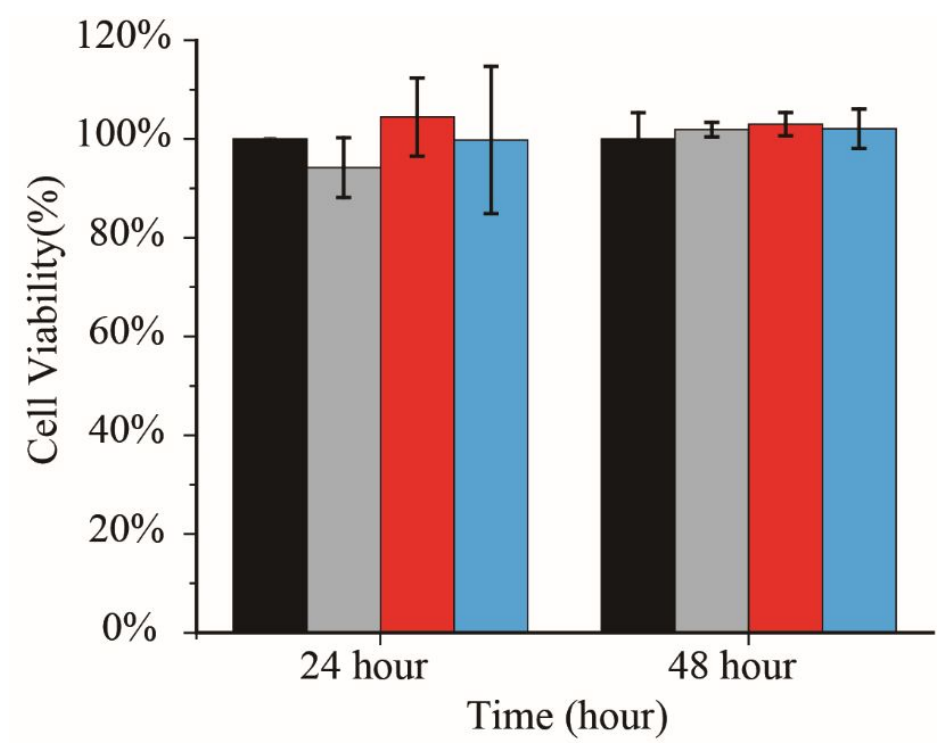

Figure S12. Cell viability of HCT116 cells treated with GCDs (grey), nanovesicles loaded with GCDs (red) and logic-gated nanovesicles loaded with GCDs (blue). HCT116 cells without treatment are used as control (black). 